\title{
Comprendre la corruption dans la governance au Cameroun
}

\author{
Richard Désiré EBELE ONANA \\ Chargé de cours \\ Université de Douala
}

\section{Résumé}

La corruption dans la governance au Cameroun est devenue un phénomène normal, et tend à se légitimer au fil des temps. Cette situation de corruption qui trouve son explication dans les comportements des agents publics et des usagers se représente sous plusieurs types et sous plusieurs formes. Dans ce pays, on se retrouve face à la grande corruption pratiquée par les acteurs du haut et à la petite corruption pratiquée par les acteurs du bas. La corruption telle que conçue et pratiquée dans la gouvernance au Cameroun, remplie plusieurs fonctions pour les acteurs de celleci qui sont à la fois politiques, économiques et sociales. La corruption dans la gouvernance au Cameroun est désormais un fait total, qui dans sa pratique, est devenue banal tant chez les usagers que chez les agents publics.

Mots clés: comprendre, corruption, gouvernance, Cameroun.

Le 17 Janvier 2017, une opération conjointe menée par les agents du Ministère des Forêts et de la Faune (MINFOF) et l'Association Last Great Apes (LAGA) a abouti, à la saisie d'une cargaison de 5040 kilogrammes (kg), environ cinq tonnes d'écailles de pangolin à Bonaberi dans la région du littoral au Cameroun. Le 18 Août de l'année 2018, une autre opération conjointe menée par les mêmes acteurs dans la même région avait conduit à la saisie de $718 \mathrm{~kg}$ de la même matière et a conduit à l'arrestation de six personnes. Un rétrospectif législatif portant sur la convention relative au commerce international des espèces de la faune et de la flore sauvages menacées d'extinction classait le pangolin géant dans la catégorie A des espèces interdites de toute commercialisation ${ }^{1}$.

La logique de conformité à ces politiques publiques de protection de certaines espèces fauniques, se traduit par la mise en place des brigades de contrôle des agents du MINFOF et la contribution d'autres forces parallèles comme la police, la gendarmerie et la douane. Pourtant, de cette affaire, il ressort que dans le premier cas, un responsable du MINFOF avait été informé de l'existence d'une telle cargaison en provenance d'une localité de la région du Sud Cameroun. Ledit responsable contactera par la suite, le délégué départemental de la zone concernée. Quelques jours plus tard, ce délégué signifiera que suite à ses recherches avec de multiples patrouilles, les résultats ont été négatifs. Dans la foulée, le même responsable va alerter le délégué régional de la région du Sud tout en lui donnant l'itinéraire empruntée par le véhicule portant la cargaison recherchée. Comme dans la précédente embuscade au niveau départemental, l'opération va se solder par un nouvel échec. Dans la poursuite de l'affaire, le responsable du MINFOF va perdre toutes les traces du chargement en question. Alors que les investigations s'intensifiaient dans la région du Sud, et aussi dans la région du Centre sans succès, deux semaines plus tard, c'est le rebondissement de l'affaire: la cargaison se trouve déjà paisiblement parquée dans une usine de production des lambris, emballée dans 300 cartons à Douala dans la région du Littoral $^{2}$. Le second cas quant à lui porte sur une cargaison en provenance de la Centrafrique qui a transité par la région du Sud et du Centre Cameroun.

Mvan le 22 Décembre 2018, dans une agence de transport Yaoundé-Douala, une foule de passagers se précipite pour prendre le bus et voyager, ceci après plusieurs heures d'attentes dues au manque de véhicules. Une femme qui, finalement vient de réussir à trouver une place dans le bus, exprime son soulagement et en même temps son inquiétude en ces mots: «merci seigneur! Enfin je suis dans la voiture pour effectuer ce voyage et fêter en famille ». Cependant, est ce possible que je puisse arriver en journée, compte tenu des arrêts qu'on fera tout le long de la route suite aux multiples contrôles de police et de gendarmerie. A la suite de cette dame, le chauffeur dudit véhicule rétorque en disant: «ma mère! Ce phénomène est très grave et nous ne savons plus quoi faire, c'est à peine si nous parvenons à verser les recettes à nos patrons. Nous avons l'impression de travailler pour ces corps de contrôle de la route. A chaque poste de police ou de gendarmerie, nous devons laisser quelque chose aux agents, que nos véhicules soient en règle ou pas. Un autre passager préoccupé par cette situation va aller plus loin en disant, « chauffeur vous voyez seulement le

\footnotetext{
${ }^{1}$ Conférence sur le commerce international des espèces de la Faune et de la Flore sauvage menacées d'extinction, tenue du 24 septembre au 5 octobre 2016 à Johannesburg.

${ }^{2}$ Entretien un responsable du MINFOF et des personnes anonymes.
} 
problème au niveau de la route, pouvez vous, vous imaginer que dans ce pays, rien ne fait plus peur à personne: Membres du gouvernement, Directeurs Généraux et autres vont en prison tous les jours pour les faits que vous decriez, mais lorsque vous entrez dans une administration publique au Cameroun, vous vous rendez compte qu'il y a comme des postes de péage partout au vu et au su de tout le monde.

A la lecture et à la compréhension de ces personnes, on se rend compte qu'elles s'insurgent contre une "perversion morale » qui laisse entrevoir la prépondérance de la corruption dans notre société, sous le regard complice ou impuissant des gouvernants.

Une analyse combinée de ces histoires brèves, nous amène à scruter la société camerounaise en nous posant la question de savoir ce qui peut expliquer autant d'actes de corruption dans presque toutes les sphères et les administrations de l'Etat au Cameroun? Ces actes de corruption manifestes, relèvent-ils de l'incompétence des agents et des choses ${ }^{3}$ ou alors des interactions collusives ${ }^{4}$ entre les usagers et les agents de l'Etat? Au regard des faits et à l'observation de la société camerounaise, la thèse des affinités sélectives et électives entre les usagers et les différents corps de contrôle ou agents de l'Etat semble plausible. En fait, il s'agit des constructions basées sur un mode d'échange illicite et accepté trivialement par tous, appelé la corruption. Entendue pour certains comme un échange occulte, secret qui permet d'accéder à des ressources que le respect des règles de procédure n'aurait pas permis d'obtenir ou aurait rendu aléatoire, et pour d'autres comme "l'abus d'une position publique en vue de l'intérêt privé »" la corruption peut être considérée comme un model d'échange privilégié des gens de l'Etat et des gens de la société ${ }^{\circ}$.

Depuis plusieurs décennies déjà, la corruption semble devenir une tendance d'échange qui se réserve toute discrimination d'échelle, de sphère et d'arène ${ }^{7}$. Pour autant qu'elle soit visible dans le public et dans le privé, elle est également perceptible dans les espaces décisionnels et opérationnels; elle se révèle être à la fois l'œuvre des dominants, autant que des dominés, et c'est en cela qu'elle prend la dimension rhizomique et se qualifie à ce titre de phénomène complexe ${ }^{8}$. Pourtant, dans ses multiples adresses à la nation, le Président de la République du Cameroun n'a cessé de faire recours à une rhétorique qui stigmatise cette pratique ${ }^{9}$. Le 3 Novembre 2004, lors de son discours de prestation de serment, il déclarait: «la fraude et la corruption se sont malheureusement infiltrées dans l'Administration publique qui devrait pourtant servir d'exemple ${ }^{10}$. Le 31 Décembre 2005, lors de son traditionnel message de nouvel an à la nation, il disait: " je ne vois que deux facteurs qui pourraient ralentir notre marche en avant. Le premier tient à l'inefficacité de certaines administrations..., mais il y a plus grave, je veux parler de la corruption que j'ai souvent dénoncée, mais qui continue à sévir. Il y a évidemment une totale incompatibilité entre les efforts que nous déployons pour faire reculer la pauvreté et l'enrichissement scandaleux de quelques-uns. Le détournement des fonds publics se fait, faut - il le rappeler, au détriment de la communauté nationale ${ }^{11}$. Le 31 Décembre 2007, Paul BIYA relevait encore: «j'ai souvent parlé des obstacles qui se dressent sur la route du progrès et qui ont pour nom, inertie administrative, fuite devant les responsabilités, intérêts personnels, laxisme et j'en passe. Mais le plus redoutable par ses conséquences et par sa difficulté à l'extirper, est sans aucun doute la corruption qui compromet notre développement et nuit à l'image de notre pays ${ }^{12}$.

\footnotetext{
${ }^{3}$ Nadine MACHIKOU, « la fabrique du « bon gouvernement »: scène constituante et instituante au Cameroun (1972-2002) », Afrilex, 2002, p. 1-34.

${ }^{4}$ Michel DOBRY., Sociologie des crises politiques, la dynamique des mobilisations multisectorielles, Paris, Presses de la Fondation Nationale des Sciences Politiques, 1992.

${ }^{5}$ Transparency International. Rapport mondial sur la corruption 2007, la corruption dans les systèmes judicaires 2007, voir en revanche l'approche de Michael JOHNSTON. «A la recherche de définition: vitalité politique et corruption », in Revue Internationale des Sciences Sociales, ${ }^{\circ}{ }^{149}$, Septembre 1996, p. 371. Selon lui, personne n’a jamais proposé une définition concise et universellement satisfaisante de la corruption.

${ }^{6}$ Luc SINDJOUN., L'Etat ailleurs entre noyau dure et case vide, Paris, Economica, 2002, p. 27.

${ }_{7}^{7}$ Jean-Pierre Olivierde SARDAN., « Le développement local comme champ politique local », in Bulletindel'APAD, 1993, pp.11-18.

${ }^{8}$ Jean-Pierre Olivierde SARDAN., «L'économie morale de la corruption en Afrique », Politique Africaine, Paris, Karthala, n 83 , octobre, 2001.

${ }^{9}$ Confère ERVING GOFFMAN, Stigmate : les usages sociaux des handicaps, Paris, les éditions de Minuit, 1975.

${ }^{10}$ Paul Biya, « Discours de prestation de serment du président de la République réélu, Cameroun tribune, 04 Novembre 2004.

${ }^{11}$ Paul Biya, «Discours du chef de 1'Etat du 31 Décembre 2005», in Cameroun tribune, Yaoundé, SOPECAM, 02 Janvier 2006.

${ }^{12}$ Paul Biya, «Discours du chef de l'Etat du 31 Décembre 2007 », in Cameroun tribune, Yaoundé, SOPECAM, 02 Janvier 2008.
} 
Au de-là de cette véhémence discursive, il a créé plusieurs institutions susceptibles de réduire la corruption et ses corollaires ${ }^{13}$ dans le champ public et privé ${ }^{14}$ du Cameroun qui cependant, peine à produire des résultats satisfaisants. Nonobstant la volonté politique de l'ordre gouvernant de réduire le leadership permissif de la corruption à travers les institutions chargées de civiliser les mœurs ${ }^{15}$ et de lutter contre ces mauvaises pratiques, la corruption reste un fait saillant qui tend à se légitimer et à s'officialiser au Cameroun. Elle est même parvenue à envahir tous les secteurs de la vie et de la société camerounaise. Si l'option de dé-civilisation et de recivilisation des mœurs ${ }^{16}$ connait un échec sournois, il faut dire que son agrégation est tributaire des rationalités centrifuges et centripètes qui fragilisent l'autocontrôle ${ }^{17}$ des agents dans une structure de jeu donnée ${ }^{18}$. On assiste alors à une configuration ${ }^{19}$ où l'échange illicite est consacré et le corrompu canonisé au détriment du refoulement des pulsions corruptives et la sacralisation des vertueux.

Prise dans cet éthos, la dynamique de surveillance et de punition ${ }^{20}$ assujettie à la logique gouverner-commanderciviliser a laissé place, à la logique d'émasculation de l'Etat ${ }^{21}$. Dès lors la «figure de l'excès et de la créativité de l'abus $\gg^{22}$ se dévoile. Pour illustrer ce point de vu, Achille MBEMBE s'appuie sur cette lettre envoyée au préfet du Wouri qui relève que: "lorsque le dossier de votre voiture s'avère complet, que vos feux-stop marchent bien, que vos clignotants aussi, puis vos phares, que votre roue de secours est gonflée, que votre extincteur flambant neuf et votre boite à pharmacie est archipleine, que le panier de la ménagère qui est à bord ne contient pas d'élément subversif... Il faut quand même vous coller un motif. Et le choix est vite fait entre "embarras sur la voie publique " et "encombrement de la chaussée »"

Dans une approche d'analyse qui se veut néo institutionnaliste, la corruption apparait comme une modalité d'échange instituée et instituante, consubstantielle à la rationalité des agents et des usagers sur scène. Comprendre donc la corruption au Cameroun, c'est établir les variables explicatives et descriptives de cette pratique sur la base des données collectées afin d'établir des liens de causalité ou de simples corrélations (I). C'est aussi étudier un fait social qui se produit de manière paradoxale dans les circuits d'échange (II).

\section{I- Les variables explicatives de la corruption au Cameroun}

Les réflexions sur la nébuleuse corruption au Cameroun peuvent s'inscrire dans une perspective de questionnement d'un fait donc la pratique est omniprésente dans toute la société. Autrement dit, la diffusion des determinants qui peuvent expliquer le fait de la corruption sont inhérente à la pénétration analytique des pratiques de ce fait dans le champ camerounais. Saisir un objet d'une telle dimension relève d'une clause de style, étant entendue que sa production peut revêtir un caractère protéiforme. A coup sûr, il s'agit de l'appropriation d'un objet dont les échelles d'action relèvent de la recomposition des logiques d'accaparement des biens publics et qui se délibère délicatement selon les dynamiques de l'esquive. A regarder de prêt, la corruption apparait comme un phénomène social total dont le réceptacle sociologique, témoigne de la discrimination persistante et précise de son ingénierie au concret.

13 Le terme «corruption» subit une surcharge sémantique en rapport avec l'entité moderne. On peut citer: Etat Kleptocratique, mafieux au sens de Jean-François BAYART dans «Fin de la partie au sud, la politique africaine de la France » in S.Michailaf, la France et l'Afrique, Paris, Karthala 1993, p.166 ; Etat prédateur dont parle Dominique DARBON dans «Etat prédateur », in Politique Africaine, n`39, 1990, pp.37-45. Etat criminel en référence à J-F BAYART, S. ELLIS, Béatrice HIBOU., « La criminalisation de l'Etat en Afrique », Paris, complexe, 1997. Etat patrimonial que parle Alain-serge Mescheriakoff. « L'ordre patrimonial essai d'interprétation du fonctionnement de l'administration d'Afrique francophone subsaharienne », in Revue française d'administration publique, n42, 1987, pp.121-149. Etat néo-patrimonial consacré par J-F Médard, «Etat d'Afrique noire », Paris, Karthala, 1991, pp.323-354.

${ }^{14}$ Pierre BOURDIEU, Le sens pratique, Paris, Minuit, 1987.

${ }^{15}$ Norbert Elias, La civilisation des mours, Paris, Calmann-Lévy, 1973.

${ }^{16}$ Nadine MACHIKOU., «Les régimes de la pacification parlementaire au Cameroun », Polis, Revue camerounaise de science politique, Vol.16, $\mathrm{n}^{\circ} 1 \& 2,2009$, pp.67-94.

${ }^{17}$ Norbert Elias, Éric DURNNING., Sport et civilisation, la violence maitrisé, Paris, Fayard, 1986.

${ }^{18}$ Michel CROZIER, Freiberg ERHARD, L'acteur et le système, les contraintes de l'action collective, Paris, Seuil, 1977.

${ }^{19}$ Norbert ELIAS, Qu'est-ce que la sociologie? , Paris, Edition du l'Aube, 1991.

${ }^{20}$ Michel FOUCAULT., Surveiller et punir, Paris, Gallimard, 1975.

${ }^{21}$ Achille MBEMBE, De la post colonie. Essai sur l'imagination politique dans l'Afrique contemporaine, Paris, 2004. p. 51.

${ }^{22}$ Achille MBEMBE, idem. p.144.

${ }^{23}$ idem., p. 173. 


\section{A- La totalisation du fait corruptif au Cameroun}

Contrairement à ce que pense Bayart ${ }^{24}$, la corruption est un phénomène visible dans toutes les sociétés du monde, mais certainement à des proportions évidemment inégales. Au Cameroun, elle a connu une agrégation sans précédent au lendemain de l'implémentation des plans d'ajustement structurel. Malgré les alertes sonnées par la société civile, les organisations non gouvernementales à l'instar de Transparency International (TI) et les associations réligieuses comme la Commission Justice et Paix de la Conférence Episcopale, le phénomène est resté frappant et assez préoccupant tant pour les agents de socialisation ${ }^{25}$, que pour les entreprises de recherche scientifique. De loin, elle est insaisissable.

Pour cause, elle prend des proportions multiples et varie en fonction du milieu. Cependant, si les premiers ne s'intéressent généralement qu'à la dimension cathartique, les seconds se tournent plutôt vers une perspective compréhensive. Dans une moindre mesure, s'inscrire dans la seconde optique, c'est ressortir les éléments causaux et ses schémas d'expression, voir son épanouissement au Cameroun.

1- Le module étiologique de la corruption et ses facteurs

Lorsque Karl MARX déclare: "A leur dire, il n'y eut jamais, l'année courante exceptée, d'autres moyens d'enrichissement que le travail et le droit. En fait, les méthodes d'accumulation primitives sont tout ce qu'on voudra, hormis matière à idylle. ${ }^{26}{ }^{2}$, il voulait démontrer que la volonté d'accumulation a toujours été au cœur des échanges. L'une des variantes de cette conception est 1 " « ethos de la manducation ${ }^{27}$ dont parle Bayart. Plusieurs causes expliquent l'existence et la résistance de ce fait social.

Alors que KLITGAARD, s'inspirant du nicaraguayen Francisco Ramirez Torres, cite les causes de la corruption en ces termes: la consommation excessive de l'alcool, les activités extraconjugales, les spéculations malheureuses, l'abus des jeux d'argent, la vanité, l'anarchie administrative, les rancœurs au sein de l'entreprise, la frustration au sein du travail, la soif de l'enrichissement illicite ${ }^{28}$, Lucien AYISSI construit une analyse autour de quatre causes: la cause métaphysique qui postule que la corruption est inhérente à la nature humaine ; la cause psychologique qui consiste pour le corrompu et le corrupteur à se soumettre aux désirs irrationnels; la cause sociopolitique et économique qui renvoie à la nature du système des valeurs et du mode de gouvernance et enfin la cause morale qui postule que la corruption est le fait de ceux qui ont réussi à aliéner leur conscience morale ${ }^{29}$.

Loin de prétendre épuiser la littérature consacrée aux causes de la corruption, il ressort qu'au Cameroun, si cette dernière trouve son sens dans les causes sus évoquées, elle puise davantage sa causalité dans la systématisation suivante:

$>$ La pauvreté mentale et matérielle. Il s'agit d'un état d'esprit dont les schèmes d'analyse déterminent une logique d'accumulation ${ }^{30}$. C'est également la précarité des conditions de vie Comme le disait Cheikh Hamidou Kane, « lorsque la main est faible, l'esprit court de grands risques $»^{31}$;

$>$ La quête du gain facile, c'est-à-dire la mise en œuvre des stratagèmes à objectif substitutionnel de biens collectifs pour un bien privé ;

$>$ Le déséquilibre salarial dans une logique comparative en terme rémunératoire, il renvoie aux frustrations et violences psychologiques dont sont victimes certains fonctionnaires causées par leurs collègues soit de la même catégorie et expérience, soit de la catégorie inférieure ;

$>$ Le chômage : c'est la résultante du faible taux de recrutement dans la fonction publique et d'un secteur privé à domination informelle;

$>$ Les lourdeurs administratives qui sont portées par le poids des procédures.

Robert KLITGAARD pose que la corruption est la résultante de la combinaison de trois facteurs: le Monopole, la Discrétion et la Responsabilité. Leur transposition se fait sur la base d'une équation mathématique structurée en $\mathrm{C}=\mathrm{M}+\mathrm{D}-\mathrm{R}^{32}$. Au Cameroun, on note les facteurs suivants:

\footnotetext{
${ }^{24}$ Jean-François BAYART., L'Etat au en Afrique. La politique du ventre, Edition Fayard, 1989. Voir aussi Pierre Péan., L'argent noir, corruption et sous-développement, Paris, Fayard, 1988.

${ }^{25}$ Confère Philippe BRAUD., Sociologie politique, $9^{\mathrm{e}}$ Edition, Paris, L.G.D.L, 2003.

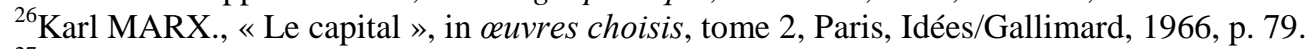

${ }^{27}$ Jean-François BAYART, Ibid., p. 69.

${ }^{28}$ Robert KLITGAARD., Combattre la corruption, Paris, Nouveaux Horizon-ARS, 1995, p. xviii.

${ }^{29}$ Lucien AYISSI., Corruption et gouvernance, Paris, L'Harmattan, 2008, pp. 97-140.

${ }^{30}$ Ebenezer NJOH MOUELLE, De la médiocrité à l'excellence, essaie sur la signification humaine du développement, quatrième édition, Clé, 2011, p. 19.

${ }^{31}$ Cheikh Hamidou Kane., L'aventure ambiguë, Paris, Union Générale d'éditions, Collection « 10/18 », 1961, p. 20.

${ }^{32}$ Robert KLITGAARD, Ibid., p.xiv
} 
$>$ Le manque de matériel dans les administrations ;

$>$ Les heures d'ouverture des administrations ;

$>$ Le manque de transparence ;

$>$ La mythologie des procédures ;

$>$ L'impunité et la mollesse des sanctions.

2- La production empiriste de la corruption

En référence aux manifestations de la corruption au MINFOF par exemple, Christophe ESSOMBA pose que, "l'épanouissement de la corruption est rendue plausible par l'utilisation abusive du marteau pour l'origine du bois, les contrôles forestiers complaisants, la falsification des déclarations sur la qualité et les quantités de bois exploités pour réduire les taxes et impôts à verser à l'Etat et aux populations riveraines,

la mise en place d'un personnel de recouvrement des taxes non agréé par la fonction publique, le versement des sommes indues lors des contrôles, la dotation abusive des bois aux autorités, l'attribution complaisante des autorisations d'exploitation forestière (privilèges accordés aux expatriés qui semblent avoir un monopole), la falsification constante des lettres de voiture, délivrances irrégulières des lettres de voiture (les gestionnaires des forêts communautaires), les fausses déclarations dans les quotas et les essences prélevées (manifestation au niveau du contrôle de document), la vente aux enchères truquées, la fraude dans la vente de gré à gré, la saisie abusive aux fins d'amener les exploitants à négocier, la production et l'acquisition des documents sécurisés au-delà de la capacité de la forêt, l'acquisition non réglementaire du quitus fiscal, la commercialisation des essences interdites d'exploitation, le blanchiment $d u$ bois (bois coupé illégalement remis sur le circuit normal), le monnayage et complaisance dans l'attribution des quitus des produits dont la gestion est régie par la CITES ${ }^{33}$ ». Dans le macrocosme sociopolitique camerounais, les manifestations de la corruption se déclinent en ces termes :

$>$ Des fraudes à l'occasion de l'organisation des concours et de recrutement d'agents dans la fonction publique, les entreprises privées, les contractuels dans les administrations publiques et parapubliques ;

$>$ L'influence des décisions rendues par la justice ;

$>$ Les rackets opérés sur les différentes voies de transport;

$>$ L'achat de conscience des citoyens par les politiciens à l'occasion des échéances électorales ;

$>$ Les violations des règles de passations de marchés publics :

$>$ Les tractations lors de dédouanement de marchandises ;

$>$ Les tractations à l'occasion de l'élaboration du fisc et du recouvrement des impôts.

La corruption s'enracine en Afrique par le truchement de trois stratégies : l'investissement de la corruption, la corruption mutualisée, la stratégie d'enrichissement rapide ${ }^{34}$.

Concernant l'investissement de la corruption, il s'agit d'une variable où le corrupteur fait des cadeaux anticipateurs à un agent public qui devient son débiteur et qui dégage l'image d'un bienfaiteur. On rentre ainsi dans la sociologie caritative où un usager ou un subordonné apporte des cadeaux à son supérieur hiérarchique. L'objectif est de créer une interdépendance tactique avec le nouveau venu. Une sorte de coopération entre égoïstes. Au Cameroun, c'est l'option privilégiée entre les élites et les nouveaux nommés d'une localité ou d'un secteur donné. En outre, l'investissement de la corruption renvoie aussi à un accueil spécial réservé à un ancien promotionnaire nouvellement affecté dans la même localité. Exemple le procureur nouvellement nommé qui est accueilli par le préfet, son ancien promotionnaire de l'Ecole Nationale d'Administration et de Magistrature (ENAM).

La mutualisation de la corruption quant à elle renvoie aux réseaux (verticaux ou horizontaux) de corruption que forment les corrompus et les corrupteurs pour une protection interne. Ce sont des alliances tissées par les agents d'un même service ou qui partageant un espace commun de travail dans l'optique de partager des gains. C'est ce qui est observable dans les contrôles mixtes policiers-gendarmerie-douane-agent du ministère des transports-agents du ministère des forêts et de la faune.

L'enrichissement rapide enfin, porte sur le recours aux moyens tous azimut permettant d'anticiper l'enrichissement sans cause et illicite d'un individu dans une société donnée. C'est-à-dire, l'exploitation rationnelle des fenêtres d'opportunité corruptive.

\footnotetext{
${ }^{33}$ Christophe ESSOMBA., Les cellules ministérielles dans la dynamique de lutte contre la corruption au Cameroun : le cas du MINFOF, mémoire, 2018, p. 75.

${ }^{34}$ KARAMOKO KANE., La corruption des fonctionnaires africains, comment sortir d'un système de capitulation générale, Yaoundé, Ed CLE, 2009, pp. 30-31. 
B- Les paramètres distinctifs de la corruption au Cameroun

Vue de loin, la corruption est insaisissable. Pour cause, elle est protéiforme et sa pratique dépend du milieu. Souvent coproduite dans des circuits et impliquant plusieurs acteurs, son appréhension est rendue possible par la systématisation de ses variables.

1- Les types de corruption

D'après KARAMOKO KANE, il existe cinq types de corruption : la corruption active et la corruption passive ; la corruption publique et la corruption privée ; la corruption nationale et la corruption internationale ; la grande et la petite corruption ; la corruption avec vol et la corruption sans vol ${ }^{35}$.

La distinction corruption active-corruption passive traduit l'idée de consubstantialité entre le corrupteur et le corrompu. La corruption active est le fait de proposer, directement ou indirectement, des avantages ou des promesses pour obtenir d'une personne dépositaire de l'autorité publique qu'elle accomplisse ou s'abstienne d'accomplir des actes de sanction. La corruption passive quant à elle intervient lorsqu'une personne dépositaire de l'autorité publique sollicite ou accepte, directement ou indirectement, des avantages pour accomplir ou s'abstenir d'accomplir des actes de sa fonction.

La corruption publique est celle dans laquelle un agent du secteur public ou parapublic est impliqué, tandis que la corruption privée concerne deux agents du secteur privé.

La corruption est nationale lorsqu'elle se passe entre deux agents du même pays. Or la corruption internationale est celle qui intervient entre des agents de pays différents.

Tandis que la grande corruption vise à changer les orientations d'un Etat pour le mettre au service d'intérêts privés, la petite corruption relève de la gestion administrative quotidienne.

La corruption se fait sans vol lorsque l'agent public obtient un pot-de-vin en plus du paiement régulier dî̀ à l'Etat. La corruption se fait avec vol dans les situations où les paiements dus au gouvernement ne sont pas effectués ou ne le sont que partiellement.

Le code pénal camerounais sur ce fait est plus intégratif et synthétique. Mention est faite ici à la corruption et à la violence électorale $^{36}$, à la corruption active ${ }^{37}$, à la corruption des agents du secteur privé ${ }^{38}$ pour ne citer que ces aspects. Cependant, la corruption active et passive mérite une délimitation précise au sens dudit code.

Dans son article 134 (1) concernant la corruption active, « est puni d'un emprisonnement de cinq (5) à dix (10) ans et d'une amende de deux cent mille (200000) à deux millions (2000000) de francs tout fonctionnaire ou agent public national, étranger ou international qui, pour lui-même ou pour un tiers, sollicite, agrée ou reçoit des offres, promesses, dons ou présents pour faire, s'abstenir de faire ou ajourner un acte de sa fonction. (2) La peine prévue à l'alinéa 1 cidessus est un emprisonnement de un (01) à cinq (05) ans et une amende de cent mille (100000) à un million (1000000) de francs si l'acte n'entre pas dans les attributions de la personne corrompue, mais a été facilité par sa fonction.

(3) Est puni des peines prévues à l'alinéa 2 ci-dessus, tout agent public national ou international qui sollicite ou accepte une rétribution en espèces ou en nature pour lui-même ou pour un tiers, en rémunération d'un acte déjà accompli ou une absence passée.

(4) Les peines prévues aux alinéas 1, 2 et 3 ci-dessus sont doublées si le fonctionnaire ou l'agent public incriminé est un Magistrat, un Officier de Police Judiciaire, un agent d'une institution de lutte contre la corruption, un Chef d'Unité administrative ou tout autre fonctionnaire ou agent public assermenté.

Quant à la corruption passive, (1) quiconque pour obtenir soit l'accomplissement, l'ajournement ou le refus d'accomplissement d'un acte, soit des faveurs ou des avantages tels que prévus à l'article 134 ci-dessus, fait des promesses, dons, présents ou cède à des sollicitations tendant à la corruption, est puni des peines prévues à l'article 134 alinéa 1 ci-dessus, que la corruption ait ou non produit son effet.

(2) Est puni des peines prévues à l'alinéa 2 de l'article 134 ci-dessus, celui qui fait des dons, présents ou cède aux sollicitations tendant à rémunérer un acte déjà accompli ou une abstention passée.

Mais, il faut distinguer les types de corruption des formes de corruption.

\footnotetext{
${ }^{35}$ KARAMOKO KANE, op cit., pp. 26-30.

${ }^{36}$ Article 123 de la loi $\mathrm{N}^{\circ} 2016 / 007$ du 12 juillet 2016 portant code pénal.

${ }^{37}$ Article 134 et 134-1 de la loi $\mathrm{N}^{\circ} 2016 / 007$ du 12 juillet 2016 portant code pénal.

${ }^{38}$ Article 312 de la loi $\mathrm{N}^{\circ} 2016 / 007$ du 12 juillet 2016 portant code pénal.
} 
2- Les formes de corruption rencontrées au Cameroun

KLITGAARD cite deux formes de corruption qui trouvent d'ailleurs leur sens au Cameroun. Il s'agit d'une part de la corruption externe qui est saisissable à partir de la rémunération de service licite, c'est-à-dire une sorte d'extorsion multiforme dont la tactique communément admise consiste à infliger à l'usager une estimation forfaitaire anormalement élevée ${ }^{39}$; la rémunération de services illicites, où l'usager graisse la patte d'un percepteur en échange d'un service, cette fois illicite, en proposant un taux trop bas ${ }^{40}$; les pots-de-vin pour couvrir une affaire et la gratification forcée pour éviter les ennuis au contribuable ${ }^{41}$. D'autre part la corruption interne, caractérisée par : le détournement des fonds par falsification des dossiers; l'impression en surnombre d'étiquettes et de timbres; la tricherie dans le recrutement du personnel ; la restitution différée des versements perçus ; la corruption des procédures d'enquêtes internes ${ }^{42}$. En outre, KARAMOKO KANE recense les actes suivants : l'abus de fonction, le blanchiment, la collusion, la concussion, le conflit d'intérêts, l'enrichissement illicite, l'entrave au bon fonctionnement de la justice, la fraude, la prise illégale d'intérêts, le recel et le trafic d'influence.

Bien que les conceptions de KLITGAARD et KANE soient transposables au Cameroun, il ressort tout de même qu'elles méritent d'être complétées par une étude de cas. Ici, est fait recours à la corruption proprement dite ${ }^{43}$ et aux infractions assimilées tels que la concussion ${ }^{44}$, le détournement de biens publics ${ }^{45}$, le trafic d'influence ${ }^{46}$, le favoritisme $^{47}$, et l'abus de fonction ${ }^{48}$.

Parmi les infractions proprement dites, on note : les extorsions, les pots de vin, l'escroquerie et le vol.

* Les extorsions

L'extorsion est le fait d'user de la menace, de la force, de la ruse ou de la contrainte pour obtenir quelque chose de quelqu'un. Ce fait est observable dans la délivrance des documents officiels aux usagers comme le permis de conduire, la carte grise ou les pièces d'identité; sur le terrain le phénomène consiste à faciliter le passage à des postes de police, de gendarmerie de douane, de forestier à des usagers en situation irrégulières sous le prétexte de mission spéciale.

* Les pots-de-vin

C'est une infraction observée dans le cadre des marchés publics et l'obtention des autorisations d'exploitation des ressources de ce secteur de la forêt et de la faune ou de l'obtention des titres fonciers dans les services de la conservation du Ministère des Domaines et des Affaires Foncières. On note aussi le paiement des sommes illégales en dehors des coûts autorisés par la loi pour obtenir une concession ou pour conclure un marché.

* L'escroquerie

C'est une pratique courante dans les différentes administrations au Cameroun. Elle renvoie au paiement de certaines sommes afin d'obtenir certains services. Ce sont aussi les rackets observés sur les routes. Le moyen utilisé est l'abus de pouvoir. Parfois les agents administratifs et de contrôle imposent certaines sommes avant de vous rendre un service. Il est de notoriété publique qu'au ministère des finances, dans les services des impôts, à la trésorerie générale et dans les autres trésoreries au Cameroun vous devez débourser entre 10 et $30 \%$ pour être servi.

* Le vol

Le vol est aussi une action qui permet à certains agents de l'Etat d'accomplir leur tâche. Par exemple, le vol du marteau forestier et des lettres de voiture au ministère des forêt et de la faune sont autant de moyens qui permettent aux agents indélicats de ce ministère non seulement d'accompagner les actes de corruption par le vol, mais aussi de blanchir le bois illégalement récolté. Parmi les infractions assimilées à la corruption ici, on relève les détournements, les blanchiments, le trafic d'influence et autres.

* Les détournements

\footnotetext{
${ }^{39}$ Voir ROBERT KLITGAARD, op cit., p. 21.

${ }^{40}$ Ibid. p. 22.

${ }^{41}$ Idem $\mathrm{p} 22$

${ }^{42}$ Idem p. 22-24.

${ }^{43}$ Article 134 du code pénal camerounais. Voir aussi NGUINI CHARLES., Plaidoyer pour un Cameroun sans corruption, Paris, L'Harmattan, 2012, pp.17-23.

${ }^{44}$ Article 137, op cit.

${ }^{45}$ Article 19, ibid.

${ }^{46}$ Article 161, ibidem.

${ }^{47}$ Article 143.

${ }^{48}$ Article 140. 
Le détournement est le fait de donner une autre direction à une ressource ou à un bien. Dans les différentes administrations publiques comme privées, ils concernent aussi bien les ressources financières que matérielles de l'Etat dans les différents services centraux et déconcentrés, le fractionnement des marchés publics, le détournement des lignes, les virements de ligne, etc. Les affaires dans le cadre de l'opération dite «Epervier» sont là pour nous le démontrer.

* Les blanchiments

Ils désignent le processus par lequel des fonds provenant d'activités illicites sont transférés dans des instruments de placement légaux ou convertis en d'autres biens dans le but d'effacer toute trace de l'origine frauduleuse ou douteuse. Ils peuvent prendre deux formes. D'une part le blanchiment des revenus issus des trafics divers qui consiste à réinvestir l'argent obtenu de manière frauduleuse, dans les transactions et collusions corruptives, dans des activités plus licites comme la construction des bâtiments, la création des plantations, le développement des activités commerciales, etc. Dans le domaine du bois par exemple, le blanchiment consiste à couper illégalement du bois sur un site, à le déplacer vers un autre site avec des lettres de voiture ou des documents permettant d'exploiter le bois dans un site différent de celui où les essences ont été recueillis.

* Le trafic d'influence

C'est le moyen par lequel les usagers réussissent à contourner les règles et la législation. Ces usagers utilisent les responsables des services centraux ou les responsables régionaux et départementaux, qui de part leurs titres, Influencent les subalternes et permettent aux usagers d'accomplir leurs activités illicites. A contrario, ces responsables supérieurs obtiennent en échange de cette aide de fortes sommes d'argent ${ }^{49}$.

\section{II- Les paradoxes explicatifs de la corruption au Cameroun}

Le regard posé sur les pratiques de corruption au Cameroun dépend souvent du bord sociopolitique où on se situe. En dépit des moyens humains, matériels et institutionnels mis en place pour réduire la corruption, son existence et sa résistance comme mobile d'interaction dépendent de la place qu'elle occupe dans l'imaginaire du citoyen. Tandis que certains trouvent en elle une pathologie sociale, d'autres en revanche la célèbre. Ces conceptions antithétiques trouvent leur sens dans les rapports officieux et officiels. Alors que la tendance répulsive s'inscrit dans des discours officiels, celle messianique gagne du terrain dans les tractations officieuses.

A- La considération pathologique de la corruption au Cameroun

Les appréhensions sur la corruption au Cameroun dépendent des acteurs, des secteurs d'activité, de la classe sociale et de l'éducation. La dimension pathologique de la corruption est une approche qui condamne la corruption dans toutes les sociétés, dans toutes ses formes parce qu'elle la considère comme une maladie. De manière structurée, il convient de les ordonner en deux catégories notamment les acteurs du haut et les acteurs du bas dont les expressions se déclinent en grande corruption et petite corruption.

1- La perception de la corruption par les acteurs du haut

Encore dénommée grande corruption, la corruption pratiquée par les acteurs du haut est un type de corruption qui concerne les personnalités venant de divers secteurs, exposées aux montants qui se chiffrent plusieurs en millions, voire en milliards. Il s'agit généralement des détournements de deniers publics ou l'acceptation des pots-de-vin dans le processus d'attribution des marchés publics. Concernant le secteur public et parapublic, les conséquences de la grande corruption sont tels que les chantiers peinent à démarrer, les retards accusés sur l'évolution des travaux dans les chantiers, les détournements des projets, la construction des éléphants blancs. La description idéale de cette situation est faite par Beatrice HIBOU lorsqu'elle parle de l' «économie de pillage ${ }^{50}$. Cet état des choses contraste avec les discours prononcés et les actions mis en œuvre pour réduire la corruption. Manifester la condamnation de la corruption par le haut, justifie la raison d'être de l'arsenal institutionnel qui avait été mis sur pieds ${ }^{51}$. Il s'agit entre autre de la Commission Nationale Anti-corruption (CONAC) $)^{52}$, du Contrôle Supérieur de l'Etat (CONSUPE) ${ }^{53}$,

\footnotetext{
${ }^{49}$ BINAM BIKOÏ C, SIPEHOUO METCHEBONG J, NTAMACK H., Etude sur l'état des lieux des pratiques de corruption dans le secteur des forêts et de la faune, Yaoundé, septembre, 2012, pp. 15-18.

${ }^{50}$ Beatrice HIBOU., « Le «Capital social » de l'Etat falsificateur ou les ruses de l'intelligence économique », in la criminalisation de l'Etat en Afrique, Paris, Edition complexe, 1997, p. 107.

${ }^{51} \mathrm{Cf}$. André TCHOUPIE, « l'institutionnalisation de la lutte contre la corruption et la criminalité financière au Cameroun »,POLIS/R.C.S. P/C.P.S.R. Vol 13, $\mathrm{n}^{\circ} 1-2,2006$, pp. 57-80.

${ }^{52}$ Créée par le décret présidentiel n²006/088 du 11 mars 2006. Ses missions sont définies dans l'article 2 dudit décret et consistent à : suivre et évaluer l'application effective du plan Gouvernemental de LCC ; recueillir, centralise et exploiter les dénonciations et informations dont elle est saisie pour les pratiques, faits ou actes de corruption ; mener toutes études ou investigations et proposer toutes mesures de nature à prévenir ou à juguler la corruption ; procéder au contrôle physique de
} 
Du Tribunal Criminel Spécial (TCS $)^{54}$, de l'Agence Nationale d'Investigation Financière (ANIF) et des Cellules Ministérielles de lutte Contre la Corruption (CMLCC) ${ }^{55}$. Mais malgré ces initiatives, l'observation constante du népotisme, du clientélisme, du néopatrimonialisme, des emplois fictifs et des détournements des deniers publics demeure.

2- La corruption vue par les acteurs du bas

Lucien AYISSI parle de «micro-politique, c'est-à-dire les rapports intersubjectifs ponctuels ou épisodiques dans le cadre de la prestation des services publics ou privés. C'est aussi celui des rapports entre groupes plus ou moins restreints de personnes liées dans le cadre des petits réseaux de corruption ${ }^{56}$. Elle est pratiquée par les cadets sociaux parce qu'elle se chiffre par des unités inferieures au million de franc CFA. Souvent omniprésente et plus stigmatisée par la société du fait d'être directement concernée, la petite corruption prend la forme de raquette, de vol et d'escroquerie. Elle est généralement visible dans les postes de contrôle : police, gendarmerie, eaux et forêt, agent des communes et du Ministère des transports ; dans certains établissements scolaires ; par les « petits » agents des services, etc. Dans une large mesure, il s'agit de la corruption qui peut être qualifiée de « corruption de survie » ou « corruption vitale ». L'objectif dans cette forme de corruption étant de compenser ou de moduler les salaires supposés faibles et insuffisants. C'est à ce titre que certains contractuels acceptent souvent les contrats qui leur sont souvent proposés dans certaines administrations notamment dans le cadre de la décentralisation.

La corruption telle que perçue par le bas est donc un appauvrissement du pauvre par le pauvre. Elle touche directement le premier parce que le dépouille de ses petites ressources au bénéfice des besoins matériels du second. Comme le relève fort pertinemment madame Carine qui se fait soutirer quelques billets de banque qu'elle trouve d'ailleurs énorme au regard de sa classe sociale, pour l'authentification d'une pièce dans une administration publique à Yaoundé ; c'est dans une perspective analogue que Monsieur Désiré se plaignait des stocks de médicament et des examens qui lui étaient demandés suite à l'accouchement de son épouse à l'hôpital. ${ }^{57}$ Parfois, l'agent corrompu opère de manière solitaire. Mais généralement, il appartient à un réseau dont il n'est qu'un élément de la chaine. Comme le rappelle Lucien AYISSI, « souvent, le chef pour lequel opère l'agent corrompu est lui aussi sous l'autorité d'un chef qui, à son tour, est sous les ordres d'un autre, et ainsi de suite. Le réseau de la corruption est, dans ce cas, une suite continue de postes de péage pour les dossiers des usagers dont le traitement est nécessairement conditionné. Le chef principal sous l'autorité duquel se déploie le réseau de la corruption est comme un dieu : « protégé par son anonymat et inaccessible, très peu de ses subalternes ne pouvant remonter jusqu'à lui, il règne sur son réseau comme une puissance invisible et terrible $»^{58}$. Comme nous l'indiquait le chef Henri, gardien de la paix que «la violence et la portion de tolérance d'un

l'exécution des conditions de passation des marchés publics ; diffuser et vulgariser les textes sur la LCC ; identifier les causes de la corruption et de proposer aux autorités compétentes des mesures susceptibles de permettre leur élimination dans tous les services publics ou parapublics ; accomplir toute autre mission à elle confiée par le président de la République.

\footnotetext{
${ }^{53}$ Voir le décret n ${ }^{\circ} 97 / 047$ du 5 mars 1997 portant organisation des services du Contrôle Supérieur de l'Etat (CSE) et le décret n ${ }^{\circ}$ 97/049 du 5 mars portant organisation du Conseil de discipline budgétaire et financière. Le CSE a compétence de mené des investigations dans l'administration, les projets ainsi que dans toutes les entreprises publiques et parapubliques. Ce dispositif de contrôle, au niveau le plus élevé de la gestion des affaires publiques est relayé par les unités techniques de contrôle interne des départements ministériels et autres organismes publics à l'effet de garantir la gouvernance économique et sociale du pays. Il a pour bras armé le CDBF dont sa mission est la prise des sanctions à l'encontre des agents publics, patent ou de fait, coupables des irrégularités et fautes ayant eu pour effet de porter préjudice aux intérêts de la puissance publique. Plus concrètement il s'agit de sanctionner les irrégularités et fautes de gestion commises par :les ordonnateurs et gestionnaires des crédits de l'Etat, des collectivités territoriales décentralisées, les entreprises et organismes publics et parapublics et toutes autre personne agissant en cette qualité ; les agents publics exerçant d'autres fonctions à titre principal, mais agissant occasionnellement ou subsidiairement comme ordonnateurs ou gestionnaires des crédits de l'Etat ; les commissaires aux comptes censeurs et commissaires de Gouvernement auprès des entreprises publiques et toute personne agissant en cette qualité.

${ }^{54}$ Voire la loi no2011/028 du 14 décembre 2011, modifiée par la loi $\mathrm{n}^{\circ}$ 2012/011 du 16 juillet 2012. Le TCS est une juridiction compétente pour connaitre des infractions de détournement des deniers publics et de corruption lorsque le préjudice est d'au moins cinquante millions de FCFA.

${ }^{55}$ Voir article portant création de la cellule ministérielle anti-corruption du MINFOF.

${ }^{56}$ Lucien AYISSI., Corruption et gouvernance, Paris, L'Harmattan, Collection « pensée africaine », 2008, p. 33.

${ }^{57}$ Source : enquêtes de terrain.

${ }^{58}$ Lucien AYISSI, op cit., p. 35. 
policier, dépendent du degré de couverture de son chef. Plus un policier sait qu'il est couvert par son chef, moins il est tolérant $»^{59}$.

On voit bien que le prolétaire subit doublement les conséquences de la corruption. D'une part indirectement par la grande corruption qui le prive des infrastructures à l'instar des routes, de l'accès aux services sociaux tels que l'eau, l'électricité, l'éducation, les hôpitaux; d'autre part, directement par les surfacturations des médicaments et le rallongement des ordonnances dans les hôpitaux, le monnayage des services publics pour échapper aux longues procédures. C'est donc de bon ton que la corruption soit rejetée en bloc par le bas bien que ce rejet dépende souvent des convenances. Ainsi, il arrive souvent qu'elle soit condamnée mais parfois considérée comme option de modulation des besoins.

B- La figure angélique de la corruption : une normalisation tendancielle de l'anormal

De la même manière que tout le monde décrie la corruption dans les discours officiels, elle est aussi acceptée par tous ou presque de manière officieuse. Lire la corruption sous le prisme sociologique consiste à démontrer la normalité de la corruption dans la société, où le pathologique relève de la condamnation ${ }^{60}$. Il s'agit d'une démarche pragmatique qu'utilisent les dominants et les dominés en interaction où justement celui qui corrompt est en règle et celui qui s'oppose à la corruption vie en marge des règles, parfois considéré comme un outsider ${ }^{61}$.

Dès lors, la corruption apparaît comme un habitus, expression des structures sociales par intériorisation de l'extériorité dans les corps et dans les têtes ${ }^{62}$.C'est une rupture totale avec la morale véhiculée dans les arènes publiques. Ici, la corruption apparaît comme un moyen pertinent, permettant de rétablir certains équilibres sociaux. C'est-à-dire un contraste entre dire et faire. Dans cet ordre d'idée, Lucien AYISSI cite cinq fonctions qui sont d'ailleurs repérables au Cameroun et permettent de bien comprendre la corruption dans cette aire géographique : les fonctions économique, politiques, sociales, psychologiques et métaphysiques ${ }^{63}$.

1- Les fonctions économiques, politiques et sociales de la corruption

En ce qui concerne la fonction économique de la corruption, il est démontré que la corruption est une opportunité permettant à toutes les classes sociales d'y participer en «captant par le bas ce que les autres prennent par le haut...Grace à la corruption, le marché serait plus un espace économique inaccessible aux pauvres, un territoire exclusivement occupé par les non-pauvres $»^{64}$. Comme le note Irène Hors «les pratiques de corruption permettent de compenser la dépendance formelle du secteur privé à l'égard de la pyramide politico-administrative par une dépendance dans les pratiques et permettant aux politiques de transférer une partie des ressources accumulées par les capitalistes aux groupes non capitalistes ${ }^{65}$. À KLITGAARD de conclure : «les versements d'argent liés à la corruption créent une sorte de mécanisme de marché. Dans un système où biens et services sont alloués en fonction des files d'attente, de la couleur politique, de choix faits au hasard ou au "mérite », la corruption peut, elle, au contraire, distribuer ces biens en fonction de l'empressement et de la capacité à payer. La corruption peut par conséquent mettre biens et services dans les mains de ceux qui les prisent le plus, qui peuvent en faire l'usage le plus efficace " ${ }^{6}$.

$\mathrm{Du}$ point de vue économique, la corruption apparait comme un outils d'intégration où on peut retrouver toutes les couches sociales, pourvu que le corrupteur ait l'échange à donner. En même temps qu'elle a la magie de faire circuler les classes sociales dans des circuits analogues, elle comble aussi le gap des procédures surabondantes de l'administration publique camerounaise. Elle passe donc de l'infortune de la bureaucratie à l'alternative d'efficacité.

La fonction politique quant à elle ouvre l'espace politique à toutes les couches sociales. Pour Lucien AYISSI, «les politiciens peuvent très bien utiliser la corruption pour favoriser l'intégration politique de diverses tribus, régions, élites ou fractions - attitude qui peut finalement être source d'harmonie face à la fragmentation de l'autorité politique,

\footnotetext{
${ }^{59}$ Source: enquête de terrain.

${ }^{60}$ Emile DURKHEIM., Les règles de méthode sociologique, Paris, PUF, 1950.

${ }^{61}$ Cf. Howard BECKER. Outsiders: études de sociologie de la déviance, Paris, Métailié, (1963) 1985.

${ }^{62}$ Pierre BOURDIEU, Le sens pratique, op cit.,

${ }^{63}$ Lucien AYISSI., Corruption et pauvreté, Paris, L’Harmattan, Collection « Pensée Africaine », 2007, pp. 47.

${ }^{64}$ Lucien AYISSI, op cit., p. 47.

${ }^{65}$ Irène HORS., « Lutte contre la corruption : Aurions-nous tort ? », in Le Courrier, N¹77 octobre-novembre 1999 , p. 75.

${ }^{66}$ Robert KLITGAARD, op cit., p. 34.
} 
aux hostilités, aux désunions ${ }^{67}$. Il s'agit du clientélisme qui est bien évidement un avatar de l'Etat occidental $l^{68}$ mais qui a pour finalité le nivellement de la classe politico-administrative camerounaise. Dans certains concours, les sommes à débourser sont connues de tous ou presque pour y accéder, bien que cela soit officieux. D'où le galvaudage systématique de technologies d'intégration comme l'équilibre régional.

Par contre, la fonction sociale de la corruption est assez illustrée par Hors. Selon elle, «la corruption contribue à résoudre ou du moins à relâcher les tensions sociales créées par le processus d'accumulation primitive »"

2- Les fonctions psychologiques et métaphysiques de la corruption

Relativement à la dimension psychologique, il ressort que la corruption libère "les pauvres du complexe d'infériorité qu'ils éprouvent dans un contexte sociopolitique où l'humanité est fonction de l'avoir et du pouvoir... Elle apaise la psychologie du pauvre qui, animé par la nécessité, l'envie et la jalousie, est capable d'une agressivité trop dangereuse pour un ordre social qui veut se protéger contre le chaos ${ }^{70}$.

La fonction métaphysique enfin apparaît dans la mesure où elle a pour finalité «la réappropriation par le pauvre de sa substance humaine... C'est l'humanisation de l'être qui ne remplissait pas les conditions pouvant lui conférer socialement le statut d'homme...Ainsi, le pauvre croit qu'il doit recourir à la corruption vitale ou défensive pour résoudre ses problèmes sociopolitiques, économiques, psychologiques et métaphysiques ${ }^{71}$.

En fin de compte, lire la corruption au Cameroun dans une perspective compréhensive, c'est d'abord admettre celle-ci comme un fait social dont, la totalisation est repérable dans les usages politique, économique et socioculturel. Ensuite, il faut la considérée comme un tremplin transactionnel, un enjeu parfois de survie dont les logiques de nivellement des déséquilibres ciblés, justifient son caractère rampant, omniprésent, multiple et multiforme. La combinaison de ces variables explicatives, détermine son emprise et sa résistance dans la société camerounaise, laquelle société tend désormais à la légitimer et l'inscrire dans la normalité du fonctionnement étatique.

\section{Bibliographie}

Achille MBEMBE., De la post colonie. Essai sur l'imagination politique dans l'Afrique contemporaine, Paris, 2004.

Alain-serge MESCHERIAKOFF, «L'ordre patrimonial essai d'interprétation du fonctionnement de l'administration d'Afrique francophone subsaharienne », in Revue française d'administration publique, n42, 1987, pp.121149.

André TCHOUPIE, « l'institutionnalisation de la lutte contre la corruption et la criminalité financière au Cameroun », POLIS/R.C.S. P/C.P.S.R. Vol 13, n 1-2, 2006, pp. 57-80.

Beatrice HIBOU., «Le « Capital social » de l'Etat falsificateur ou les ruses de l'intelligence économique », in la criminalisation de l'Etat en Afrique, Paris, Edition complexe, 1997.

BINAM BIKOÏ C, SIPEHOUO METCHEBONG J, NTAMACK H., Etude sur l'état des lieux des pratiques de corruption dans le secteur des forêts et de la faune, Yaoundé, septembre, 2012, pp. 15-18.

Cheikh Hamidou Kane., L'aventure ambiguë, Paris, Union Générale d'éditions, Collection « 10/18 », 1961.

Christophe ESSOMBA., Les cellules ministérielles dans la dynamique de lutte contre la corruption au Cameroun : le cas du MINFOF, mémoire, 2018, Inédit.

Dominique DARBON., « Etat prédateur », in Politique Africaine, n³9, 1990, pp.37-45.

Emile DURKHEIM., Les règles de méthode sociologique, Paris, PUF, 1950.

Erving GOFFMAN, Stigmate : les usages sociaux des handicaps, Paris, les éditions de Minuit, 1975.

Howard BECKER., Outsiders : études de sociologie de la déviance, Paris, Métailié, (1963) 1985.

Irène HORS., « Lutte contre la corruption : Aurions-nous tort ? », in Le Courrier, N 177 octobre-novembre 1999, p. 75.

Jean-François BAYART., «Fin de la partie au sud, la politique africaine de la France » in S.Michailaf, la France et l'Afrique, Paris, Karthala 1993.

Jean-François BAYART., L'Etat au en Afrique. La politique du ventre, Edition Fayard, 1989.

Jean-François Médard, «Etat d'Afrique noire », Paris, Karthala, 1991, pp. 323-354.

Jean-Pierre Olivier de SARDAN., «Le développement local comme champ politique local », in Bulletin de l'APAD, 1993, pp.11-18.

\footnotetext{
${ }^{67}$ Lucien AYISSI, ibid., p. 49.

${ }^{68} \mathrm{Cf}$. Luc SINDJOUN, op cit.

${ }^{69}$ Irène HORS., ibid., p. 75.
} 
Jean-Pierre Olivier de SARDAN., "L'économie morale de la corruption en Afrique », Politique Africaine, Paris, Karthala, n83, octobre, 2001.

Jean-François BAYART, S. ELLIS, Béatrice HIBOU., « La criminalisation de l'Etat en Afrique », Paris, complexe, 1997.

KARAMOKO KANE., La corruption des fonctionnaires africains, comment sortir d'un système de capitulation générale, Yaoundé, Ed CLE, 2009.

Karl MARX., «Le capital », in œuvres choisis, tome 2, Paris, Idées/Gallimard, 1966.

La loi N²016/007 du 12 juillet 2016 portant code pénal.

Luc SINDJOUN., L'Etat ailleurs entre noyau dure et case vide, Paris, Economica, 2002,

Lucien AYISSI., Corruption et gouvernance, Paris, L’Harmattan, Collection « pensée africaine », 2008.

Lucien AYISSI., Corruption et pauvreté, Paris, L'Harmattan, Collection « Pensée Africaine », 2007.

Michael JOHNSTON., «A la recherche de définition : vitalité politique et corruption », in Revue Internationale des Sciences Sociales, $\mathrm{n}^{\circ} 149$, Septembre 1996.

Michel CROZIER, Friedberg ERHARD., L'acteur et le système, les contraintes de l'action collective, Paris, Seuil, 1977.

Michel DOBRY., Sociologie des crises politiques, la dynamique des mobilisations multisectorielles, Paris, Presses de la Fondation Nationale des Sciences Politiques, 1992.

Michel FOUCAULT., Surveiller et punir, Paris, Gallimard, 1975.

Nadine MACHIKOU, « la fabrique du « bon gouvernement » : scène constituante et instituante au Cameroun (19722002)», Afrilex, 2002, pp. 1-34.

Nadine MACHIKOU., «Les régimes de la pacification parlementaire au Cameroun », Polis, Revue camerounaise de science politique, Vol.16, $\mathrm{n}^{\circ} 1 \& 2,2009$, pp.67-94.

NGUINI CHARLES., Plaidoyer pour un Cameroun sans corruption, Paris, L’Harmattan, 2012.

Norbert Elias, Éric DURNNING., Sport et civilisation, la violence maitrisé, Paris, Fayard, 1986.

Norbert Elias., La civilisation des mœurs, Paris, Calmann-Lévy, 1973.

Norbert ELIAS., Qu'est-ce que la sociologie ?, Paris, Edition du l'Aube, 1991.

Philippe BRAUD., Sociologie politique, 9e Edition, Paris, L.G.D.L, 2003.

Pierre BOURDIEU., Le sens pratique, Paris, Minuit, 1987.

Pierre PEAN., L'argent noir, corruption et sous-développement, Paris, Fayard, 1988.

Robert KLITGAARD., Combattre la corruption, Paris, Nouveaux Horizon-ARS, 1995.

Transparency International. Rapport mondial sur la corruption 2007, la corruption dans les systèmes judicaires 2007. 